Mediterránea Ser. Biol. (1988), n. ${ }^{\circ}$ 10. Pág. 13-18

\title{
LIBERACIÓN DEL C,N Y P DURANTE LA DESCOMPOSICIÓN DE LA PAJA DEL ARROZ
}

\author{
por
E. FORÉS', M. MENENDEZ ', F. A. COMÍN ${ }^{1}$
}

\section{RESUMEN}

La paja del arroz enterrada en el sedimento al finalizar la cosecha, se descompone con una tasa instantánea de descomposición de 0.00702 día-1. Al cabo de los 139 días que el arrozal permanece seco, queda el $48 \%$ de la biomasa, el $31.9 \%$ del fósforo y el $38.9 \%$ del nitrógeno iniciales. El proceso de descomposición de la paja aporta el $33 \%$ del nitrógeno total y el $8 \%$ del fósforo total incorporados por la actividad humana al cultivo.

\section{ABSTRACT}

Rice straw buried in the sediment of a rice-field during the dry season decomposes at a rate of 0.00702 day- $1.48 \%$ biomass, $32 \%$ phosphorus and $39 \%$ nitrogen remain after 139 days decay. Rice straw decomposition can furnish $33 \% \mathrm{~N}$ and $8 \% \mathrm{P}$ of the total provided by in the Ebro Delta.

\section{INTRODUCCIÓN}

Enterrar la paja del arroz es una práctica habitual en el cultivo del arroz por inundación. Con esta operación se pretende recicla el contenido de nutrientes del suelo para la próxima cosecha. A pesar de su importancia, existen escasos trabajos sobre la contribución que esta actividad agrícola representa en el balance de elementos nutritivos en los arrozales (ACHARYA, 1935, a, b; MIRANDA 1963).

Las tasas de descomposición del material vegetal varían en función de diferentes factores. La concentración inicial de nutrientes es uno de ellos. Se ha observado, por ejemplo, que hojas con un elevado contenido en nitrógeno se descomponen más rápidamente que las que poseen un contenido menor. (BO-

(1) Departament d'Ecologia. Facultat de Biologia. Universitat de Barcelona. Diagonal, 645. 08028 BARCELONA. 
COCK, 1963; KAUSHIK y HYNES, 1971; HOWARTH y FISHER, 1976). Por su parte los detritívoros consumen preferentemente detritus vegetal en avanzado estado de descomposición con una relación C: $\mathrm{N}$ baja y abundante recubrimiento bacteriano. (CUMMINS ET AL, 1973; KAUSHIK Y HYNES, 1971). Otros factores, como la temperatra, también pueden variar la tasa de descomposición (GOSZ ET AL, 1973b).

En este trabajo se presentan los resultados de una experiencia realizada en un arrozal del Delta del Ebro para conocer la dinámica de liberación de nutrientes carbono, nitrógeno y fósforo durante el proceso de descomposición de la paja del arroz. Asimismo se discute la importancia de este proceso en la disponibilidad de nutrientes en los arrozales.

\section{MATERIAL Y MÉTODOS}

El cultivo del arroz en el Delta del Ebro tiene lugar desde abril hasta principios de octubre. La paja de arroz utilizada en la experiencia se recolectó después de la cosecha, y se cortó en fragmentos de 6 a $8 \mathrm{~cm}$. de longitud. Fragmentos equivalentes a 2.6-4.7 g. de peso seco se depositaron en el sedimento del arrozal en bolsas de nylon de dos tamaños de malla, $2 \mathrm{~mm}$. y $100 \mathrm{um}$. Se recolectaron tres réplicas de cada tamaño de malla al cabo de $1,3,15,30,60$, 120 y 139 días del inicio de la experiencia (3 de noviembre de 1986). La última recolección se realizó pocos días antes de la incorporación del abono de fondo.

Las muestras se trasladaron al laboratorio donde se llevaron a cabo las determinaciones de peso seco y homogeneizado para analizar el contenido en cenizas, carbono, nitrógeno (analizador elemental) y fósforo total (JACKSON, 1970). Todas las determinaciones se realizaron por duplicado.

La técnica de depositar una cantidad de material vegetal en bolsas de tamaño de malla reducido posee el inconveniente de infraestimar la descomposición debida a los macroconsumidores, pero minimiza la pérdida de pequeños fragmentos formados durante el proceso (GOSZ ET AL, 1973 a).

\section{RESULTADOS}

Las diferencias observadas en la disminución de la biomasa entre las bolsas de malla gruesay fina no son significativas $(\mathrm{p}<0.001) \mathrm{Al}$ cabo de un mes de incubación la biomasa de la paja del arroz era $67.9 \%$ de la biomasa inicialmente depositada en las bolsas. A los 139 días quedaba únicamente el $37.7 \%$ (Fig. 1).

La tasa instantánea de descomposición (k) para la paja del arroz calculada según el modelo exponencial propuesto por OLSON (1963) para describir la disminución de biomasa debida a procesos biológicos durante la descomposición, es de 0.00702 día-1. El tiempo necesario para reducir la biomasa en un $50 \%$, es de 99 días. En el período de 139 días de incubación se observa un incremento del porcentaje de cenizas del 16.6 al $30 \%$ del peso seco total.

La variación del contenido en carbono, nitrógeno y fósforo durante la descomposición tampoco muestra diferencias significativas $(p<0.01)$ entre las bolsas de malla fina y gruesa. Se observa una rápida liberación de nutrientes durante los primeros quince días, especialmente para el fósforo $(38.9 \%$ del ini- 

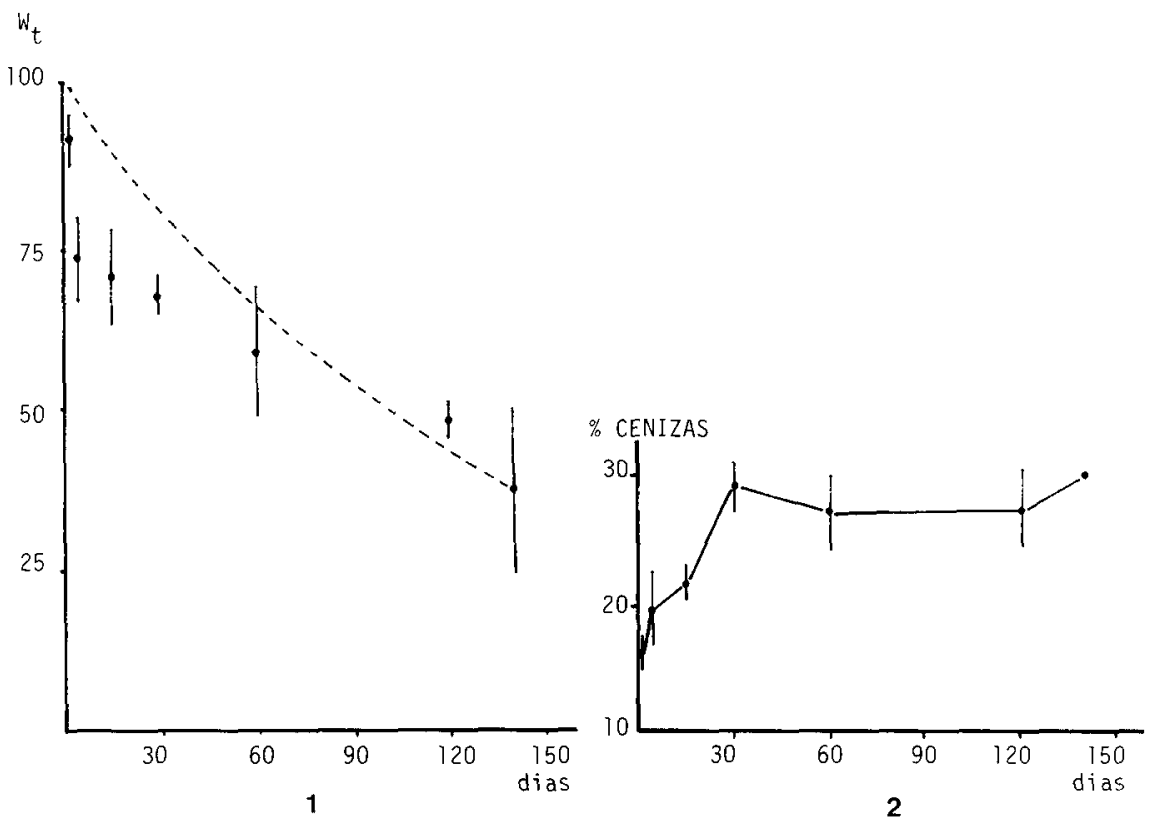

Figura 1.- Tanto por ciento de la biomasa inicial, durante el proceso de descomposición. Las líneas verticales indican la desviación estandard. La línea punteada muestra la descomposición teórica a partir del modelo exponencial de Olson (1963).

Figura 2.- Contenido de cenizas de la paja del arroz durante el proceso de descomposición.

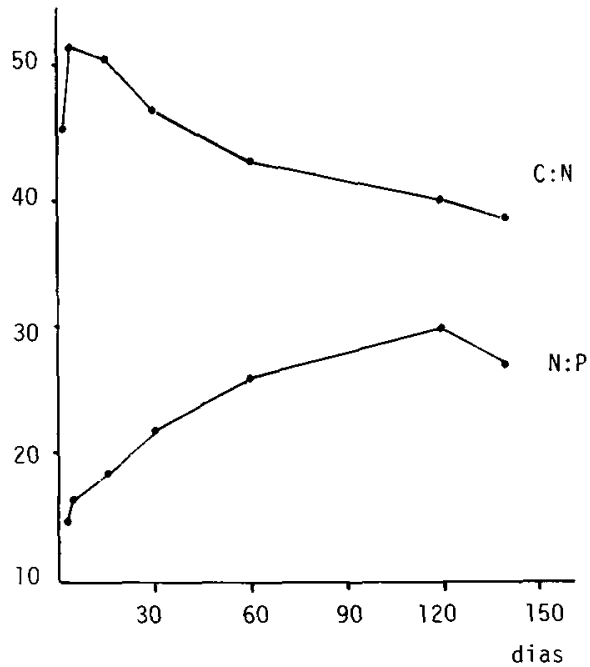

Figura 3.- Relaciones C:N y N:P de la paja del arroz durante el proceso de descomposición. 
cial). Al final del proceso se han liberado el $78.1 \%$ y el $61.1 \%$ del fósforo y nitrógeno total iniciales, respectivamente. La abundancia relativa en la liberación de nutrientes sigue el orden $\mathrm{N}<\mathrm{C}<\mathrm{P}$. La relación $\mathrm{C}$ : $\mathrm{N}$ y $\mathrm{P}$ pasa de $41: 1$ y 678:1 a 39:1 y 1035:1, respectivamente. La relación $\mathrm{N}: \mathrm{P}$ se incrementa de 15:1 a 27:1 durante el mismo período de estudio (Fig. 3).

Las tasas de pérdida de biomasa y nutrientes para cada intervalo de tiempo, $\mathrm{k}=\left(\ln \left(\mathrm{w}_{\mathrm{o}} / \mathrm{wt}\right) / \mathrm{t}\right) \cdot 100$ (HOWARTH-WILLIAMS \& DAVIES 1979) se presentan en la tabla 1 . Se observa que el carbono y el nitrógeno tienen unas tasas diarias de pérdida similares a la de la biomasa. Las tasas de pérdida más elevadas para el fósforo se observan durante los primeros tres días. Después va disminuyendo, pero suele ser superior a la del resto de nutrientes estudiados.

\section{DISCUSIÓN}

La pérdida de biomasa de la paja durante el proceso de descomposición se desvía de la curva teórica calculada considerando que el proceso es regido por sistemas biológicos y por tanto controlado enzimáticamente. (SAUNDERS, 1976). La diferencia entre curva teórica y real, tal como sucede en nuestro estudio, se observa en muchos estudios de descomposición de material vegetal, (NICHOLS y KEENY, 1973; HOWARD-WILLIAMS y DAVIES, 1979; NEWELL, FELL y MILLER, 1986; BASTARDO, 1979; MEYER, 1980; ATTIWILL, 1968; KAUSHIK y HYNES, 1971), interpretándose como difusión de material soluble durante la lisis celular. Por ello, las diferencias más notables se producen durante los tres primeros días. Durante estos días se observó una gran abundancia de oligoquetos en las bolsas de descomposición, no observada durante el resto del proceso. Su presencia podría estar relacionada con el incremento de bacterias degradadoras de azúcares y proteoliticas, observados en este tipo de experiencias (BASTARDO, 1979).

\begin{tabular}{crrrr} 
Días & \multicolumn{1}{c}{ p.s. } & \multicolumn{1}{c}{$\mathrm{C}$} & \multicolumn{1}{c}{$\mathrm{N}$} & $\mathrm{P}$ \\
\hline $0-1$ & 8.30 & 10.21 & 23.45 & 31.05 \\
$1-3$ & 11.33 & 13.19 & 9.52 & 20.96 \\
$3-15$ & 0.30 & 0.26 & 1.66 & 0.43 \\
$15-30$ & 0.90 & 0.27 & 0.00 & 2.55 \\
$30-60$ & 0.50 & 0.00 & 0.00 & 0.08 \\
$60-120$ & 0.35 & 0.50 & 0.40 & 0.11 \\
$120-139$ & 1.20 & 1.48 & 1.90 & 1.29 \\
\hline
\end{tabular}

Tabla 1.-Tanto por ciento diario de la tasa de descomposición (k), calculada a partir de la siguiente expresión; $k=\left(\ln \left(w_{0} / w t\right) / t\right) .100$, donde $w_{o}$ representa el $100 \%$ de la biomasa inicial, wt el porcentaje de biomasa inicial presente después de thoras de incubación. Se ha calculado la $\mathrm{k}$ para peso seco, carbono, nitrógeno y fósforo. 
Según ATTIWILL (1968) y GOSZ AT AL (1973 b), si la liberación de un nutriente es paralela a la pérdida de peso seco es porque el elemento en cuestión está, en gran proporción formando parte de la estructura molecular de la pared vegetal celular, la más resistente a la descomposición. Éste sería nuestro caso para el carbono y nitrógeno, según los resultados expresados en la tabla 1. La pérdida más rápida para el fósforo se explicaría por una mayor proporción de este elemento en forma soluble. En nuestro caso, el material soluble ya ha sido translocado en gran parte a la espiga durante su formación (Forés y Comín, datos no publicados).

Aportes de nutrientes $\mathrm{N}$ total $(\mathrm{kg} / \mathrm{ha}) \quad \mathrm{P}$ total $(\mathrm{kg} / \mathrm{ha})$

\begin{tabular}{llr} 
Descomposición de la paja & 31.05 & 1.85 \\
Abonado fondo + foliar & 62.15 & 20.29 \\
TOTAL & 93.20 & 22.14 \\
\hline
\end{tabular}

Tabla 2.-Aportes humanos de nitrógeno y fósforo al cultivo.

La falta de diferencias significativas entre las bolsas de malla gruesa y fina, así como la falta de organismos pertenecientes a la mesofauna en las bolsas de malla gruesa hacen pensar que la descomposición por consumo no tiene una gran importancia en los arrozales, por lo que respecta al periodo seco en que tiene lugar (octubre a abril). En su lugar la descomposición bacteriana durante la inundación y la fúngica durante los meses de invierno cobrarían mayor importancia (YONEYAMA \& YOSHIDA, 1977).

Según los datos obtenidos en nuestra experiencia, la descomposición de la paja del arroz (tallo y hojas), aporta el $33.3 \%$ del nitrógeno y el $8.4 \%$ del fósforo de los totales de nitrógeno y fósforo aportados regularmente al cultivo por el hombre (Tabla 2). Aquí no se consideran los aportes correspondientes a la raíz $(3000 \mathrm{Kg} / \mathrm{ha}$ con un contenido inicia de $0.87 \%$ de $\mathrm{N}$ y $0.12 \%$ de $\mathrm{P}$ ) ni la descomposición que puede tener lugar durante la inundación de los campos, aunque las condiciones anaeróbicas presentes la hagan más lenta. (ACHARYA, a, b 1935; REICE, 1974). 


\section{BIBLIOGRAFIA}

ACHARYA, C. N. (1935a). Studies on the anaerobic decomposition of plant materials. I. The anaerobic decomposition of rice straw (O. sativa). Biochem. J. 29: 528-541.

- (1935b). Studies on the anaerobic decomposition of plant materials. II. Comparison of the course of decomposition of rice straw under anaerobic, aerobic and partially aerobic conditions. Biochem. J. 29: 1116-1120.

ATTIWILL, P. M. (1968). The lost of elements from decomposing litter. Ecology 49: 142-145. BASTARDO, H. (1979). Laboratory studies on decomposition of littoral plants. Pol. Arch. Hydrob. 26 (3): 257-256.

BOCOCK, K. (1963). Changes in the amount of nitrogen in decomposing leaf litter of sessile oak (Querqus petraea) J. Ecol. 51: 555-566.

CUMMINS, K. M.; R. C. PETERSEN; F. O. HOWARD; J. C. WUYCHECK \& V. I. HOLT (1973). The utilization ofleaf litter by stream detritivors. Ecology 54: 336-345.

GOSZ, J. R.; G. E. LIKENS \& F. H. BORMANN (1973a). Nitrogen release from decomposing leaf and branch litter in the Hubbard Brook Forest, New Hampshire. Ecol. Monogr. 43: 173-191.

- (1973b). Nutrient content of litter fall on the Hubbard Brook Experimental Forest. New Hamphire. Ecology. 53 (5): 769-784.

HOWARD-WILLIAMS, C. \& B. R. DAVIES (1979). The rates of dry matter and nutrient loss from decomposing Potamogeton pectinalis in a brakish south-temperate coastal take. Fresh. Biol. 9: 13-21.

HOWARTH R. W. \& S. G. FISHER (1976). Carbon, Nitrogen and Phosphorus dynamic during leaf decay in nutrient-enriched stream microecosystem. Fresch. Biol. 6: 221-228.

JACKSON, M. L. (1970). Análisis químico del suelo. Ed. Omega. Barcelona. 662 pp.

KAUSHIK N. K.\& H. B. H. HYNES (1971). The fate of dead leaves that fall into the streams. Arch. Hydrob. 60 (4): 465-515.

MEYER, J. L. (1980). Dynamic of phosphorus and organic matter during leaf decomposition in a forest stream. Oikos 34: 44-53.

MIRANDA, V. B. (1963). Organic manuring and incorporation of residues for rice. Agrochimica. 8: $17-24$.

NEWELL, S. Y.; J. W. FELL \& C. MILLER (1986). Deposition and decomposition of Turtlegrass leaves. Int. Rev. Ges. Hydrob. 71 (3): 363-369.

NICHOLS, D. S. \& D. R. KEENY (1973). Nitrogen and phosphorus release from decaying Water Milfoil. Hydrobiol. 42 (4): 509-525.

OLSON, J. S. (1963). Energy storage and the balance of producers and decomposers in ecological systems. Ecology 44 (2): 322-331.

REICE, S. R. (1974). Environmental patchiness and the breakdown of leaf litter in a woodland stream. Ecology 55: 1271-1282.

SAUNDERS, G. W. (1976). Decomposition in freshwater. In The role of terrestrial and aquatic organisms in decomposition processes. J. M. Anderson and A. Macfadyen. Ed. pp.: 341373. Blackwell Scientific Publications. Oxford.

YONEYAMA, T. \& T. YOSHIDA (1977). Decomposition of rice residue in tropical soils. II Inmobilization of soil and fertilizer nitrogen by intact rice residue soil. Soil. Sci. Plant Nutr. 23 (1): $41-48$. 